\title{
Synergistic scale inhibition of both IA/AMPS copolymer and magnetic field
}

\author{
Zhan Liu ${ }^{1,2}$, Meifang $\mathrm{Yan}^{1,2}, \mathrm{Na} \mathrm{Li}^{3}$, Yuhu Gao ${ }^{1,2}$, Haihua $\mathrm{Li}^{1,2}$, Zhenfa Liu ${ }^{1,2}$ \\ ${ }^{1}$ Institute of Energy Resources, Hebei Academy of Science, Shijiazhuang Hebei 050081, China; \\ ${ }^{2}$ Hebei Engineer Research Center for Water Saving in Industry, Shijiazhuang Hebei 050081, China \\ ${ }^{3}$ Hebei Sun-water Treatment Co., LTD., Shijiazhuang Hebei 050081, China
}

\begin{abstract}
The antiscaling rate of both water magnetic device and IA/AMPS copolymer was investigated by using a stationary scale inhibition experimental device, and the result illustrated that there was a synergistic scale inhibition effect between magnetic field and IA/AMPS copolymer. Under the condition the experimental water through the water magnetizer was $120 \mathrm{~L} / \mathrm{h}$, and the magnetic field strength was $0.7 \mathrm{~T}$, the magnetization time of $40 \mathrm{~min}$, the antiscaling rate of both water magnetic device and copolymer IA/AMPS to $\mathrm{CaCO}_{3}$ increased $11 \%$ as compared with that of the copolymer IA/AMPS alone. At this time, the dosage of copolymer IA/AMPS was $6 \mathrm{mg} / \mathrm{L}$. And the synergistic antiscaling rate of both water magnetic device and IA/AMPS copolymer to $\mathrm{Ca}_{3}\left(\mathrm{PO}_{4}\right)_{2}$ increased $25 \%$ as compared with that of the copolymer IA/AMPS alone under the same condition except dosage of IA/AMPS copolymer was $20 \mathrm{mg} / \mathrm{L}$.
\end{abstract}

\section{Introduction}

In the industrial circulating water system, it is generally necessary to add scale inhibitors to achieve the effect of scale inhibitors and corrosion inhibitors. Phosphate inhibitors have been widely used due to their good scale inhibitors[1-2]. However, the large-scale use of phosphate scale inhibitors will cause water quality nutrient enrichment, promote a large number of bacteria and algae derivatives, and seriously affect the ecological environment[3]. Itaconic acid belongs to a wide range of biologically fermented products and has received widespread attention[4]. AMPS is a water-soluble monomer containing sulfonic acid groups, which have strong hydrophilicity, can enhance the solubility of copolymer, and can make copolymer scale inhibitor have good inhibition, complexation, adsorption and dispersion of calcium phosphate scale and iron oxide, etc.In addition, the introduction of sulfonic acid groups can enhance the rigidity and thermal stability of the polymer molecular chain, so that the polymer has good temperature resistance[5].

Magnetized water treatment technology has the advantages of simple operation, low operating cost, non-toxic and pollution-free. Magnetized water treatment technology is a very promising scale inhibition technology from both environmental and economic perspectives. But when the treatment method is applied alone, the effect is not ideal.

In this paper, Itaconic acid was copolymerized with 2-acrylamido-2-methylpropanesulfonic acid (AMPS) which contain sulfonic acid group to obtain an IA /
AMPS copolymer. Then, a comprehensive physical and chemical treatment method was used to synergize the magnetized water treatment technology with the IA/AMPS copolymer to study its synergistic scale inhibition performance[6]. The purpose is to reduce the amount of IA/AMPS copolymers and develop a new process for the magnetic field-water treatment agents to co-process industrial circulating cooling water with low operating cost.

\section{Experimental}

\subsection{Chemicals and experiment apparatus}

\subsubsection{Chemicals}

Itaconic acid (IA), distilled water, Sodium hydroxider solution with a mass fraction of $30 \%$, 2-acrylamido-2-methylpropane sulfonic acid (AMPS), ammonium persulphate, sodium hydrogen sulfite, calcium chloride, etc., all of the drugs are analytically pure are analytical grade.

\subsubsection{Experiment apparatus}

HW.SY11-KP3 electrically heated thermostatic water bath, Beijing Changfeng Instrument Co., Ltd.;Dual-beam UV-visible spectrophotometer, Shanghai Yuan Analytical instruments Co., Ltd.; HWCL-3 constant temperature electromagnetic stirrer, Zhengzhou Great Wall Technology Industry \& Trade Co., Ltd.; Magnetic water device (self-restraint), etc. 
We made a magnetized treatment equipment by ourselves. The schematic diagram of magnetic water device is shown in Fig. 1.

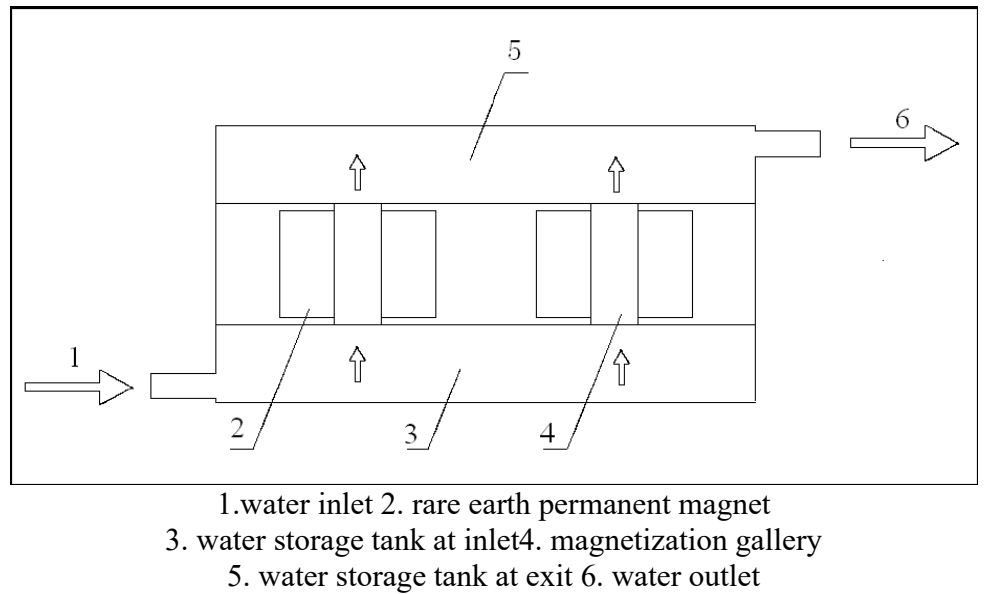

Fig. 1. Schematic diagram of homemade water magnetic device

\subsection{Synthesis of the copolymer IA/AMPS}

Typically, a given amount of IA and AMPS were added into a four-necked flask which contained a thermometer and a condenser, and then put some distilled water, isopropanol and ammonium persulfate into the four-necked flask. Then place the flask in a thermostatic water bath with a magnetic stirrer. When the temperature of the liquid reaches our desired value, a certain concentration of hydrogen peroxide and sodium bisulfite solution were added dropwise using two syringe pumps. Make sure that the two agents are added in the same time. And then it reacted at a constant temperature for a period of time. The product was left at room temperature, and when the reactant solution was cooled to room temperature, the solution was discharged. The obtained pale yellow transparent liquid was an aqueous polymer solution.

\subsection{Scale inhibition rate by static method of the copolymer IA/AMPS and the synergistic properties with magnetic fields}

\subsubsection{Scale inhibition rate by static method of IA / AMPS polymer to $\mathrm{CaCO}_{3}[7]$}

The experiment used mixed water and non-evaporative concentration to determine the resistance to $\mathrm{CaCO}_{3}$ (the concentration of calcium ion in the solution was $600 \mathrm{mg} \cdot \mathrm{L}^{-1}$, and the concentration of bicarbonate ion was $\left.1200 \mathrm{mg} \cdot \mathrm{L}^{-1}\right) . \quad 500 \mathrm{~mL}$ of the formulation water containing the polymer agent was heated to $80^{\circ} \mathrm{C}$, and then the temperature maintained at $80^{\circ} \mathrm{C}$ for 10 hours. Then it was taken out and cooled to room temperature. Titrate with EDTA solution and calculate the calcium ion content in the liquid supernatant after cooling, and do a blank experiment at the same time. The rate of scale inhibition is calculated by equation 1 .

$$
\text { Rate of scale inhibition }=\left(\frac{C_{1}-C_{0}}{C_{2}-C_{0}}\right) \times 100 \%
$$

Equation 1. The calculation formula of scale prevention rate

$\mathrm{C}_{0}$ represents the mass concentrations of $\mathrm{Ca}^{2+}$ in the blank water sample after heated. $\mathrm{C}_{1}$ represents the mass concentrations of $\mathrm{Ca}^{2+}$ in the water sample with the inhibitor after heated. And $\mathrm{C}_{2}$ stands for unheated formulated water.

\subsubsection{Measurement of the rate of static scale inhibition on $\mathrm{Ca}_{3}\left(\mathrm{PO}_{4}\right)_{2}$}

The experiment used mixed water and non-evaporative concentration to determine the resistance to $\mathrm{Ca}_{3}\left(\mathrm{PO}_{4}\right)_{2}$ (The concentration of calcium ion in the solution was $250 \mathrm{mg} \cdot \mathrm{L}^{-1}$ and the concentration of phosphate ion was $150 \mathrm{mg} \cdot \mathrm{L}^{-1}, \mathrm{pH}$ was equal to 9.0 (Adjust with $25 \mathrm{~g} \cdot \mathrm{L}^{-1}$ sodium borate solution)). $500 \mathrm{~mL}$ of the formulation water containing the polymer agent was heated to $80^{\circ} \mathrm{C}$, and then maintained at $80^{\circ} \mathrm{C}$ for 10 hours. Then it was taken out and cooled to room temperature. The $\mathrm{PO}_{4}{ }^{3-}$ content in the supernatant after cooling was measured with a 721 spectrophotometer, and a blank experiment was performed at the same time. The rate of scale inhibition is calculated by equation 2 .

$$
\text { Rate of scale inhibition }=\left(\frac{C_{1}-C_{0}}{C_{2}-C_{0}}\right) \times 100 \%
$$

Equation 2.C alculation formula of scale prevention rate

$\mathrm{C}_{0}$ represents the mass concentrations of $\mathrm{PO}_{4}{ }^{3-}$ in the blank water sample after heated. $\mathrm{C}_{1}$ represents the mass concentrations of $\mathrm{PO}_{4}{ }^{3-}$ in water sample with polymer medicament after heated. And $\mathrm{C}_{2}$ stands for unheated formulated water. 


\section{Results and Discussion}

\subsection{Scale inhibition of the polymer IA / AMPS under the synergy of the magnetic field}

The scale inhibition rate to $\mathrm{CaCO}_{3}$ and $\mathrm{Ca}_{3}\left(\mathrm{PO}_{4}\right)_{2}$ of using the copolymer alone and its synergy with water magnetizer at different IA/AMPS copolymer concentrations were investigated when the flow rate of the experimental water through the water magnetizer was $120 \mathrm{~L} / \mathrm{h}$, and the magnetic field strength was $0.7 \mathrm{~T}$, the magnetization time of $40 \mathrm{~min}$. The results are shown in Fig.2. and Fig.3.

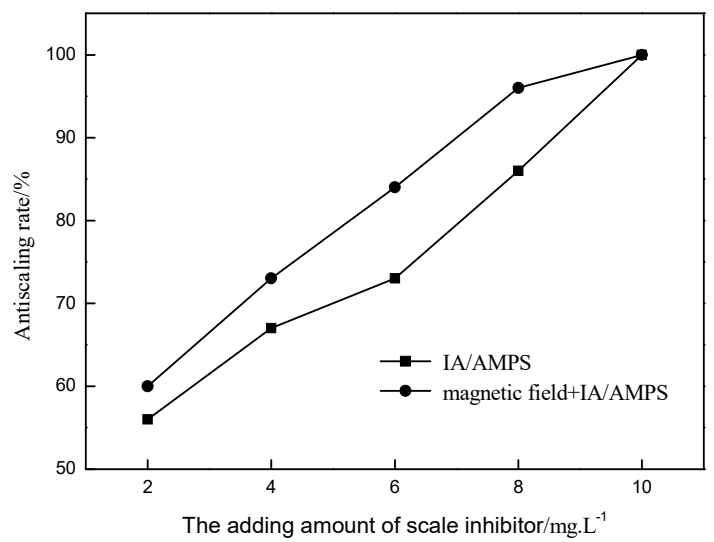

Fig. 2. The antiscaling rate of the polymer IA/AMPS under the magnetic field to $\mathrm{CaCO}_{3}$

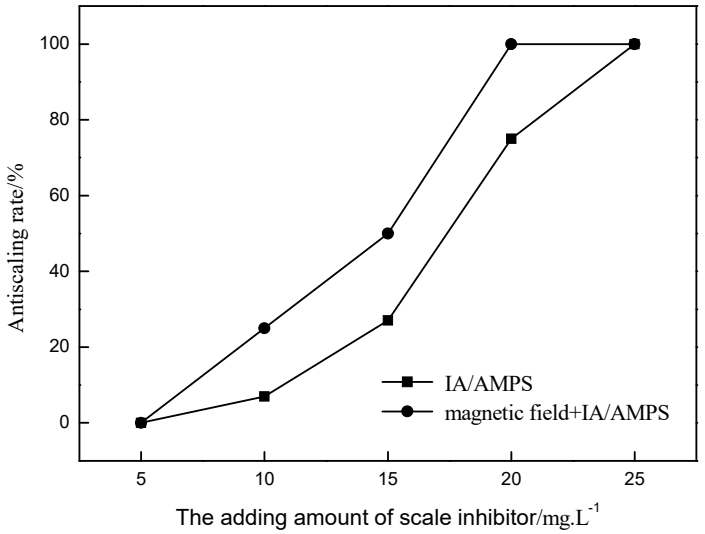

Fig. 3. The antiscaling rate of the polymer IA/AMPS under the magnetic field to $\mathrm{Ca}_{3}\left(\mathrm{PO}_{4}\right)_{2}$

Fig.2 can show that the antiscaling rate to calcium carbonate when magnetic field and IA / AMPS copolymer work together. When the water flow of the prepared water through the water magnet was $120 \mathrm{~L} / \mathrm{h}$, and the magnetic field strength was $0.7 \mathrm{~T}$, the magnetic treatment time of $40 \mathrm{~min}$, and an amount of IA / AMPS copolymer was $6 \mathrm{mg} / \mathrm{L}$, the scale ratio was $11 \%$ higher than that of the polymer IA / AMPS alone.

From Fig.3, it is shown that the addition of the magnetic field greatly improves the scale inhibition rate. Under the same conditions, the antiscaling rate to $\mathrm{Ca}_{3}$ $\left(\mathrm{PO}_{4}\right)_{2}$ by passing the water magnetic device and copolymer IA/AMPS together was increased $25 \%$ except that the amount of IA / AMPS copolymer was $20 \mathrm{mg} / \mathrm{L}$.

\subsection{Mechanism analysis}

(1) In summary, the above experimental results show that the polymer IA / AMPS has good scale inhibition and dispersion properties. This is because when the IA /
AMPS copolymer is dissolved in water, it will ionize and generate negatively charged molecular chains. These negatively charged molecular chains can chelate with calcium ions to form complexes that are soluble in water. Then the complex makes the dissolving ability of the scale-forming compound increase, thereby acting as a scale inhibitor.

(2) The addition of a magnetic field improves the scale inhibition rate to a certain extent. This is because that under normal circumstances, calcium ions and anion ions in water all exist as hydrated ions. However, when the hydrated ions flow through the magnetic field, they will be subject to Lorentz force to make a spiral circular motion, and the positive and negative ions rotate in opposite directions. This situation will destroy the physical hydration layer of the ions, and under sufficient magnetic field strength, the chemical hydration layer of the ions will also be destroyed. In this way, these scaling ions are released from the hydrated state and become "naked" ions. Then the opportunity for direct collision 
between the released calcium ions and the anions to combine to form a compound increases, thereby forming a large number of compound nuclei and microcrystals. These microcrystals are mainly formed in water, and the particles are finely dispersed and easy to be carried away by the water flow, thereby greatly reducing the chance of scaling on the pipe wall.

\section{Conclusion}

The above experiments show that both the copolymer IA/AMPS and the water magnetic device can prevents scaling to calcium carbonate and calcium phosphate. The best conditions for the synergistic effect of the magnetic field and the IA/AMPS copolymer on calcium carbonate scales are the water flow of the prepared water through the water magnet $120 \mathrm{~L} / \mathrm{h}$, the magnetic field strength of $0.7 \mathrm{~T}$, the magnetic treatment time of $40 \mathrm{~min}$. When the dosage of the agent was $6 \mathrm{mg} / \mathrm{L}$, the antiscaling rate of the water magnetic device and the copolymer to calcium carbonate increased significantly, an increase of $11 \%$. Under the same conditions, when the dosage of the agent was $20 \mathrm{mg} / \mathrm{L}$, the scale inhibition rate on calcium phosphate can be increased by $25 \%$ when the magnetic field and the agent cooperate.

The magnetization and water treatment agents have a good synergistic scale inhibition effect. The addition of a magnetic field can effectively improve the scale inhibition performance of water treatment agents and has a wide application prospect.

\section{Acknowledgements}

This work was financially supported by the science and technology projects of Hebei Academy of Sciences (21712).

\section{References}

1. ROOMI Y A, HUSSEIN K F, RIAZI M R. (2012) Inhibition efficiencies of synthesized anhydride based polymers as scale control additives in petroleum production, Journal of Petroleum Science and Engineering, 81(1): 151-160.

2. Shao H, Wang Y, Zhou Y, Leng Y.X, et al. (2015) Preparation and Inhibition Performance of IA-HPEC Copolymer Seale Inhibitor, Acta Petrolei Sinica (Petroleum Processing Section), 31(03) : 769-775.

3. HASSON D, SHEMER H, SHER A, (2011) State of heart of friendly "green" scale control inhibitors; review article, Industrial \& Engineering Chemistry Research, 50 (12) : $7601-7607$.

4. Li P, Han Z.C, Zhao S.L, Li F. (2015) Research on the synthesis of scale inhibitor copolymer IA/MA/MAA/AMPS and its scale inhibition capacity. Industrial Water Treatment, 35(07):47-50.

5. Zhang A.Q, Zhang G.H,Wei H, Li L.J. (2011) Preparation and performance of a new sulfonate copolymer scale inhibitor, Chemical Industry and Engineering Progess, 30(08):1858-1861.

6. Liu Z.F, Gao Y.H, Zhang .H, et al.(2008) Synergistic scale inhibition of polyepoxysuccinic acid with magnetic field and the study of scale inhibition mechanism. Fine Chemicals,25(10): 1003-1007.

7. Liu.Z, Yan M.F, Liu Z.F, Li N.(2019) Synthesis and properties of the IA/AMPS copolymer, IOP Conference Series, Earth and Environmental Science, 330(042049): 1-6. 